\title{
A Class of Stationary Solutions of the Einstein-Maxwell Equations
}

\author{
W. B. Bonnor \\ Queen Elizabeth College, University of London, London, U.K.
}

Received April 27, 1973

\begin{abstract}
The class depends on one harmonic function and two additional arbitrary constants. It refers to sources with spin and electric or magnetic charge, and includes some space-times which are flat at spatial infinity. However, it does not include a solution for a spinning particle with monopole charge and mass.
\end{abstract}

\section{§1. Introduction}

Among the known electrostatic solutions of Einstein-Maxwell theory are those of Papapetrou-Majumdar (PM) [1,2] and those of Weyl [3]. Members of the PM class need have no spatial symmetry but every source is such that, in relativistic units,

$$
m=|e|,
$$

$m$ and $e$ being the mass and charge. Weyl's solutions have axial symmetry but the sources are less specialised and satisfy

$$
m=k e,
$$

$k$ being a constant, the same for all. Thus the two classes are different, but have some common members.

Recently the PM solution has been generalised to what are called PIW solutions $[4,5]$. These are stationary, need have no spatial symmetry, and arise from sources satisfying

$$
m=|e|, \quad \boldsymbol{h}= \pm \boldsymbol{\mu},
$$

$\boldsymbol{h}, \boldsymbol{\mu}$ being angular momentum and magnetic moment. It is a natural step to seek that class of axially symmetric solutions which is related to the PIW class in a way similar to that in which the Weyl class is related to the PM class. The solutions would depend on two harmonic functions (like PIW) and would have

$$
m=k e, \quad \boldsymbol{h}=k^{\prime} \boldsymbol{\mu} .
$$


In the course of an unsuccessful search for such solutions I found a more restricted class, satisfying (1.2) but different from Weyl's class. These will be described in this paper. They depend on one harmonic function and refer to electric sources with spin, with an accompanying magnetic field. By choice of the harmonic function one can make the metric Minkowskian at spatial infinity. However, the class contains no realistic solution for massive sources, and in this respect resembles the rotating solutions of Papapetrou [6].

The field equations used in this work are

$$
\begin{gathered}
R_{\mu v}=-8 \pi E_{\mu v}, \\
4 \pi E_{v}^{\mu}=-F^{\mu \alpha} F_{v \alpha}+\frac{1}{4} \delta_{v}^{\mu} F^{\alpha \beta} F_{\alpha \beta}, \\
F_{\mu v ; \sigma}+F_{v \sigma ; \mu}+F_{\sigma \mu ; v}=0, \\
F_{; v}^{\mu v}=J^{\mu}=0,
\end{gathered}
$$

where $E_{v}^{\mu}$ is the electromagnetic energy tensor, $F_{\mu v}$ the electromagnetic field tensor, and $J^{\mu}$ the four-current which vanishes because we consider only the exterior field. Greek indices range from 1-4, and Roman indices from 1-3. A semi-colon denotes covariant differentiation with respect to the metric of space time, and a comma denotes partial differentiation.

\section{$\S 2$. The Solution}

We start with the stationary axially symmetric metric in the form

$$
d s^{2}=-f^{-1}\left\{f e^{\lambda}\left(d z^{2}+d r^{2}\right)+r^{2} d \alpha^{2}\right\}+f(d t-w d \alpha)^{2},
$$

where $f, \lambda$ and $w$ are functions of $z$ and $r$. The main problem is to find $f$ and $w$, since, given these, $\lambda$ can be determined by integration. The electromagnetic field $F_{\mu \nu}$ arises from two scalar potentials $\phi$ and $\psi$ as follows $[4,5]$

$$
\begin{aligned}
F_{4 n} & =\phi_{, n}, \\
F^{m n} & =f \gamma^{-\frac{1}{2}} \varepsilon^{m n p} \psi_{, p},
\end{aligned}
$$

$\gamma$ being the determinant of the three dimensional metric in the curly bracket of (2.1), namely

$$
\gamma=f^{2} e^{2 \lambda} r^{2}
$$

and $\varepsilon^{m n p}$ being the permutation symbol with values \pm 1 and 0 . 
The Maxwell equations (1.7), (1.8) and the Einstein-Maxwell (EM) equations (1.5), (1.6) then give

$$
\begin{gathered}
\nabla^{2} \psi=f^{-1}\left(f_{1} \psi_{1}+f_{2} \psi_{2}\right)+r^{-1} f\left(w_{2} \phi_{1}-w_{1} \phi_{2}\right) \\
\nabla^{2} \phi=f^{-1}\left(f_{1} \phi_{1}+f_{2} \phi_{2}\right)+r^{-1} f\left(w_{1} \psi_{2}-w_{2} \psi_{1}\right) \\
\nabla^{2} w-2 r^{-1} w_{2}=-2 f^{-1}\left(f_{1} w_{1}+f_{2} w_{2}\right)+4 r f^{-2}\left(\psi_{1} \phi_{2}-\psi_{2} \phi_{1}\right) \\
\nabla^{2} f-f^{-1}\left(f_{1}^{2}+f_{2}^{2}\right)=2\left(\phi_{1}^{2}+\phi_{2}^{2}+\psi_{1}^{2}+\psi_{2}^{2}\right)-r^{-2} f^{3}\left(w_{1}^{2}+w_{2}^{2}\right),
\end{gathered}
$$

where suffices 1 and 2 mean differentiation with respect to $z$ and $r$ respectively, and $\nabla^{2}$ is the Laplacian in cylindrical polar coordinates. The EM equations also determine $\lambda$ up to an additive constant by means of

$$
\begin{aligned}
\lambda_{1}=-f^{-1} f_{1}+r f^{-2} f_{1} f_{2}-4 r f^{-1}\left(\phi_{1} \phi_{2}+\psi_{1} \psi_{2}\right)-r^{-1} f^{2} w_{1} w_{2}, \\
\lambda_{2}=-f^{-1} f_{2}+\frac{1}{2} r f^{-2}\left(f_{2}^{2}-f_{1}^{2}\right)+2 r f^{-1}\left(\phi_{1}^{2}-\phi_{2}^{2}+\psi_{1}^{2}-\psi_{2}^{2}\right) \\
+\frac{1}{2} r^{-1} f^{2}\left(w_{1}^{2}-w_{2}^{2}\right) .
\end{aligned}
$$

These equations satisfy $\lambda_{12}=\lambda_{21}$ if (2.4)-(2.7) are satisfied, and they are consistent with the remaining non-trivial field equation

$$
\lambda_{11}+\lambda_{22}=r^{-1} f^{-1} f_{2}-\frac{1}{2} f^{-2}\left(f_{1}^{2}+f_{2}^{2}\right)+\frac{1}{2} r^{-2} f^{2}\left(w_{1}^{2}+w_{2}^{2}\right) .
$$

The Eqs. (2.4)-(2.7) have been put into a compact form by Ernst [7], but for my purposes it is more convenient to leave them as they are. Other workers who have studied them are Neugebauer and Kramer [8], and Harrison [9].

To derive the new solution we suppose that $\psi$ and $f$ are both functions of $\phi$, and that $w$ is related to $\phi$ as follows:

$$
\psi=\psi(\phi), \quad f=f(\phi), \quad w_{1}=r h(\phi) \phi_{2}, \quad w_{2}=-r h(\phi) \phi_{1},
$$

and that none of the functions $\psi, f$ or $h$ is a constant.

The condition $w_{12}=w_{21}$ requires that $\phi$ satisfy

$$
\nabla^{2} \phi+h^{-1} h^{\prime}\left(\phi_{1}^{2}+\phi_{2}^{2}\right)=0 \text {, }
$$

where ' means $d / d \phi$. Eq. (2.6) is now satisfied identically and (2.4), (2.5) and (2.7) give respectively

$$
\begin{gathered}
\nabla^{2} \phi+\left(\frac{\psi^{\prime \prime}}{\psi^{\prime}}-\frac{f^{\prime}}{f}+\frac{f h}{\psi^{\prime}}\right)\left(\phi_{1}^{2}+\phi_{2}^{2}\right)=0, \\
\nabla^{2} \phi-\left(f h \psi^{\prime}+f^{-1} f^{\prime}\right)\left(\phi_{1}^{2}+\phi_{2}^{2}\right)=0, \\
\nabla^{2} \phi+\left[\frac{f^{\prime \prime}}{f^{\prime}}-\frac{f^{\prime}}{f}+\frac{f^{3} h^{2}-2\left(\psi^{\prime 2}+1\right)}{f^{\prime}}\right]\left(\phi_{1}^{2}+\phi_{2}^{2}\right)=0 .
\end{gathered}
$$


These three equations must all be the same as (2.12). From (2.13) and (2.14) we find

$$
\psi^{\prime \prime}+f h\left(1+\psi^{\prime 2}\right)=0
$$

and from (2.12) and (2.14),

$$
f h \psi^{\prime}+f^{-1} f^{\prime}+h^{-1} h^{\prime}=0 .
$$

Eqs. (2.16) and (2.17) give

$$
\frac{\psi^{\prime} \psi^{\prime \prime}}{1+\psi^{\prime 2}}=\frac{f^{\prime}}{f}+\frac{h^{\prime}}{h}
$$

which may be integrated to yield

$$
f h+k\left(1+\psi^{\prime 2}\right)^{\frac{1}{2}}=0
$$

$k$ being an arbitrary constant. Substituting for $f h$ from (2.19) into (2.16) we obtain an equation for $\psi$ :

$$
\psi^{\prime \prime}-k\left(1+\psi^{\prime 2}\right)^{\frac{3}{2}}=0 .
$$

We now introduce a new variable $v(\phi)$ by means of

$$
\psi^{\prime}=\tan v
$$

and so obtain from $(2.20)$

$$
\frac{d v}{d \phi}=k \sec v
$$

whence, on integration

$$
\phi=k^{-1} \sin v+\phi_{0},
$$

$\phi_{0}$ being an arbitrary constant. Further, (2.19) gives

$$
f h=-k \sec v \text {, }
$$

and, using (2.22) in (2.21) and integrating, we have

$$
\psi=-k^{-1} \cos v+\psi_{0}
$$

$\psi_{0}$ being another constant. Equating (2.14) and (2.15), substituting for $\psi^{\prime}$ and $f h$ from (2.21) and (2.24), we get a second order differential equation for $f$ of which the solution is

$$
f=k^{-2}(2+a \sin v+b \cos v),
$$

$a$ and $b$ being further arbitrary constants. From this and (2.24) we obtain

$$
h=-\frac{k^{3} \sec v}{2+a \sin v+b \cos v} .
$$


From (2.12) it follows that the function $\chi(\phi)$ given by

$$
\chi=\int h d \phi
$$

is harmonic, i.e. satisfies

$$
\nabla^{2} \chi=0,
$$

and we find from (2.27), (2.28) and (2.22) that

$$
\chi=-k^{2} \int(2+a \sin v+b \cos v)^{-1} d v .
$$

There are different cases of this integral, which are discussed in the next section. Here we collect together the formulae which constitute our solution:

$$
\left.\begin{array}{rl}
\phi & =k^{-1} \sin v+\phi_{0}, \\
\psi & =-k^{-1} \cos v+\psi_{0}, \\
f & =k^{-2}(2+a \sin v+b \cos v), \\
w_{1} & =-r f^{-1} v_{2}, \quad w_{2}=r f^{-1} v_{1}, \\
\lambda_{1} & =-f^{-1} f_{1}+r k^{-4} f^{-2}\left(a^{2}+b^{2}-4\right) v_{1} v_{2}, \\
\lambda_{2} & =-f^{-1} f_{2}-\frac{1}{2} r k^{-4} f^{-2}\left(a^{2}+b^{2}-4\right)\left(v_{1}^{2}-v_{2}^{2}\right) ;
\end{array}\right\}
$$

$v$ is a function of $r, z$ given in terms of a harmonic function $\chi$ by $(2.30), \lambda_{1}$ and $\lambda_{2}$ are obtained from (2.8) and (2.9), the electromagnetic field from (2.2) and (2.3), and the metric from (2.1). The solution therefore depends on one harmonic function and five arbitrary constants, of which only two have physical importance, as we shall see.

\section{§ 3. Physical Interpretation}

There are three different cases of the integral in (2.30) according as $a^{2}+b^{2} \gtreqless 4$. If $a^{2}+b^{2}=4$ my solution is a special case of PIW and one can then choose $\phi_{0}$ and $\psi_{0}$ in (2.31) so that

$$
f=\phi^{2}+\psi^{2} \text {. }
$$

If $a^{2}+b^{2} \neq 4$ one has

$$
f=\text { const }+\phi^{2}+\psi^{2}, \quad(\text { const } \neq 0) .
$$

I shall examine in detail only the case $a^{2}+b^{2}<4$ : the others are not physically very different. The integral on the right hand side of (2.30) is

$$
-\frac{2 k^{2}}{\left(4-a^{2}-b^{2}\right)^{\frac{1}{2}}} \tan ^{-1}\left[\frac{(2-b) \tan \frac{1}{2} v+a}{\left(4-a^{2}-b^{2}\right)^{\frac{1}{2}}}\right]+\text { const , }
$$


and absorbing multiplicative and additive constants into the harmonic function $\chi$ we may write

Now put

$$
\tan \frac{1}{2} v=(2-b)^{-1}\left[-a+\left(4-a^{2}-b^{2}\right)^{\frac{1}{2}} \tan \chi\right] .
$$

$$
\chi=\theta+c
$$

where $c$ is a constant and $\theta$ a harmonic function; then by choosing $c$ so that

we obtain

$$
\tan c=a\left(4-a^{2}-b^{2}\right)^{-\frac{1}{2}}
$$

Let us now examine the solution at infinity, where $\theta$ is assumed to vanish at least like $R^{-1}\left(R^{2}=z^{2}+r^{2}\right)$. Then (3.6) gives for sufficiently large $R$

$$
v \sim \frac{2(2+b)}{\left(4-a^{2}-b^{2}\right)^{\frac{1}{2}}} \theta \text {. }
$$

As $R \rightarrow \infty$ we wish $f$ in (2.31) to tend to unity so we choose

$$
2+b=k^{2}
$$

(since in the case under consideration $b^{2} \leqq 4$, this implies $k^{2} \leqq 4$ ). We can now write down the asymptotic form for large $R$ of the solution (2.31). We put $\phi_{0}$ zero and $\psi_{0}$ equal to $k^{-1}$, we absorb a factor $2(2+b)^{\frac{1}{2}}$ $\left(4-a^{2}-b^{2}\right)^{-\frac{1}{2}}$ into $\theta$, and then obtain

$$
\left.\begin{array}{rl}
\phi & \sim \theta, \\
\psi & \sim \frac{1}{2} k \theta^{2}, \\
e^{-\lambda} & \sim f \sim 1+a k^{-1} \theta, \\
w_{1} & \sim-r k \theta_{2}, \quad w_{2} \sim r k \theta_{1},
\end{array}\right\}
$$

$\theta$ being a harmonic function which tends to zero at infinity at least like $R^{-1}$, but is otherwise arbitrary. This shows how the solution depends on two physically significant arbitrary constants $a$ and $k$, in addition to $\theta$.

If we choose $\theta=e / R$ to obtain the electric field of a point charge, (3.8) gives $w \sim$ const $+e k z / R$ and we obtain an unrealistic source of angular momentum. (Actually it is a semi-infinite massless line source along the $z$ axis as in the NUT solution [10].)

We next try a dipole potential $\theta=\mu z / R^{3}$, and obtain for the asymptotic form of the quantities in (3.8):

$$
\left.\begin{array}{rl}
\phi & \sim \mu z / R^{3}, \\
\psi & \sim \mu h z^{2} / R^{6}, \\
e^{-\lambda} & \sim f \sim 1-2 z d R^{-3}, \\
w & \sim-2 h r^{2} / R^{3},
\end{array}\right\}
$$


where we have put $2 h=k \mu, 2 d=-a \mu / k$. This solution refers to a point source of angular momentum $h$, with electric dipole moment $\mu$. It has no mass, and unless $d$ is put zero it carries a mass dipole. The electric dipole apparently does not rotate, otherwise there would be a magnetic quadrupole field, which is absent. The leading term in the magnetic potential does nevertheless arise from the interaction of the electric dipole and angular momentum terms. It is of order $R^{-4}$ and does not occur in Maxwell's theory.

\section{§4. Conclusion}

The Eqs. (2.4)-(2.7) are invariant under a duality rotation, that is, if $(\phi, \psi)$ are a solution with given $(f, w, \lambda)$ so are $[\phi \cos \beta-\psi \sin \beta$, $\phi \sin \beta+\psi \cos \beta]$ where $\beta$ is a constant. Hence the solution of $\S 3$ can at once be applied to a magnetic instead of an electric source (by putting $\beta=\pi / 2$ ), or to a linear combination of the two.

Physically the solutions are of limited value because a realistic spinning source can be obtained only by ruling out a monopole mass source. Perhaps the most interesting feature is the unexpected magnetic potential, visible in (3.9), which arises from the spin-electric field interaction.

\section{References}

1. Papapetrou,A.: Proc. Roy. Irish Acad. 51, 191 (1947)

2. Majumdar, S. D.: Phys. Rev. 72, 390 (1947)

3. Weyl, H.: Ann. Physik 54, 117 (1917)

4. Perjés, Z.: Phys. Rev. Letters 27, 1668 (1971)

5. Israel, W., Wilson, G. A.: J. Math. Phys. 13, 865 (1972)

6. Papapetrou, A.: Ann. Physik VI F. 12, 309 (1953)

7. Ernst, F. J.: Phys. Rev. 168, 1415 (1968)

8. Neugebauer, G., Kramer, D.: Ann. Physik VII F. 24, 62 (1969)

9. Harrison, B. K.: J. Math. Phys. 9, 1744 (1968)

10. Bonnor, W. B.: Proc. Cambridge Phil. Soc. 66, 145 (1969)

W. B. Bonnor

Queen Elizabeth College

University of London

Campden Hill Road

London, W. 8., U.K. 
